\title{
Energetic potential of plant biomass and its use
}

\author{
Michael Ioelovich \\ Dept.of Chemistry, Designer Energy Ltd, Rehovot, Israel
}

Email address:

bd895892@zahav.net.il (M. Ioelovich)

\section{To cite this article:}

Michael Ioelovich. Energetic Potential of Plant Biomass and Its Use, International Journal of Renewable and Sustainable Energy. Vol. 2, No. 2, 2013, pp. 26-29. doi: 10.11648/j.ijrse.20130202.11

\begin{abstract}
A calorific value of various biomass samples (waste of wood, paper, textile, agricultural plants) and liquid biofuels (bioethanol, biodiesel) has been studied in this paper. In addition, a specific combustion heat of the biomass samples was calculated, which almost was coincided with the experimental heating value. It was established that the specific combustion heat of the investigated solid plant materials was from 11 to $25 \mathrm{MJ} / \mathrm{kg}$. The calorific value of the liquid biofuels was from 27 to $37 \mathrm{MJ} / \mathrm{kg}$, i.e. it was higher than the calorific value of the solid biomass samples. Despite of this fact, a most efficient way of the energy production is the direct burning of the plant biomass, while the burning of such amount of the liquid biofuel, which can be obtained from the plant material, gives a much smaller energetic effect.
\end{abstract}

Keywords: Biomass, Biofuel, Combustion Heat

\section{Introduction}

Nowadays, the non-renewable fossil resources - coal, petroleum and gas, are the main energy sources, which cover more than $80 \%$ of the world's energy needs [1]. A considerable attention in recent years is given to alternative energy sources, and especially to plant biomass, which in contrast to the fossil fuels is continuously renewed in the nature. As a result of the biosynthesis, a mass of the plant biomass increases approximately on 1.5 trillion tons annually [2]. Actually, only small part of the biomass can be utilized. The share of the biomass has about $10 \%$ of the world energy consumption [3].

Various biomass samples and especially wastes of forest, wood, textile, pulp and paper, agriculture and cities, as well as some plant species can be used as a feedstock for production of biofuels. Total amount of such raw materials, which is accumulated in USA only, is about 1 billion tons annually. The plant biomass can be utilized directly as a solid fuel or after its conversion into liquid biofuel, such as bioethanol or biodiesel [4].

To choose the most efficient way for production of the bioenergy, it is necessary to know the energy potential of the biomass samples. Thus, the main purpose of this paper was to determine the calorific value of various types of biomass in comparison with the energetic capacity of the liquid biofuels isolated from these plant materials.

\section{Experimental}

\subsection{Materials}

Various biomass types were used and studied:

Sawdust of poplar Populus Canadensis

Agricultural waste, such as wheat and rice straw, bagasse of sugar cane, corn stover, fallen olives fruits and olive pomace

Used newsprint and printing papers, as well as used cardboard

Waste of cotton and textile industry

Residual lignin isolated from poplar wood by acid hydrolysis [5]

- Residue remaining after enzymatic hydrolysis of the biomass

- Pure cotton cellulose (Hercules Inc)

The biomass samples were preliminary milled in a lab "Waring"- mill, screened through a sieve to obtain the fraction with particle size of $1-3 \mathrm{~mm}$ and then dried at $110^{\circ} \mathrm{C}$ up to constant weight.

In addition, the following liquid biofuels were studied:

Olive oil (Olivio Ltd)

Modified biodiesel that was produced by transesterification of olive oil with methanol according to the procedure [6]

Bioethanol that was obtained by fermentation of the hydrolyzate from the enzymatically hydrolyzed biomass [7, 8]. The fermentation was carried out with the yeasts of Sac- 
charomyces cerevisiae [9] in the laboratory fermentor "Biostat A Plus" (Sartorius AG). Distillation of the diluted ethanol was performed repeatedly in a vacuum evaporator at $60^{\circ} \mathrm{C}$, until the $95 \%$ ethanol concentration.

\subsection{Methods}

The chemical composition of plant materials was determined by conventional methods of chemical analysis $[5,7]$. The content of total carbohydrates was measured after delignification of the biomass with sodium chlorite. To remove the hemicelluloses, the obtained sample was hydrolyzed with boiling $1.5 \%$ hydrochloric acid for $2 \mathrm{~h}$. The content of cellulose was calculated from the dry residue remained after hydrolysis of the total carbohydrates. Content of lignin was determined by means of standard TAPPI procedure T222, while content of ash in accordance with TAPPI standard T211. Three samples of the same biomass were tested to calculate an average content of the component and standard deviation. The standard deviation at determination of percentage of the component was in the range $\pm 1 \%$.

The standard enthalpy or higher heating value (E) of dehydrated samples $(1 \mathrm{~g})$ was determined in the bomb calorimeter "Parr $6400^{\prime}$ at $25^{\circ} \mathrm{C}$. The net (lower) specific combustion heat $(\mathrm{Q})$ was calculated by the equation:

$$
\mathrm{Q}=\mathrm{E}-0.225 \mathrm{H}
$$

where $\mathrm{H}$ is percentage of hydrogen in the sample.

To obtain accurate results, three samples of the same biomass were burned. The standard deviation didn't exceed $\pm 0.5 \mathrm{MJ} / \mathrm{kg}$.

\section{Results and Discussion}

Results of the chemical analysis of the dried plant materials were showed in Table 1 . The greatest amount of cellulose $(95-98 \%)$ was in the residues of textile and cotton industry, while the highest amount of lignin (33-37\%) was observed for the olive pomace and residues after enzymatic hydrolysis of wood biomass. Some waste samples, such as rice straw, used newsprint and printing paper contained an increased amount of ash (16-29\%). Other components of the biomass samples were hemicelluloses and extractive substances.

Table 1. Chemical composition of dry biomass samples*.

\begin{tabular}{llll}
\hline Biomass sample & Cel. \% & Lig. \% & Ash, \% \\
\hline Cotton linter & 5 & 1 & \\
Waste of textile & 8 & 0 & \\
Used printing paper & 1 & 1 & 9 \\
Used newspaper & 8 & 21 & 8 \\
Used cardboard & 8 & 10 & 2 \\
Sawdust of wood & 4 & 23 & \\
Bagasse & 8 & 20 & \\
Corn stover & 37 & 19 & 6 \\
\hline
\end{tabular}

\begin{tabular}{llll}
\hline Biomass sample & Cel. \% & Lig. \% & Ash, \% \\
\hline Wheat straw & 5 & 16 & \\
Rice straw & 5 & 10 & 6 \\
$\begin{array}{l}\text { Olive pomace } \\
\text { Residue }\end{array}$ & 5 & 33 & \\
of hydrolysis & 2 & 37 & \\
\hline
\end{tabular}

*Note: Cel is Cellulose, Lig is Lignin.

The study of the energetic potential of various biomass samples showed that the high-lignified samples and the lignin itself exhibited the greatest specific combustion heat of 21.0-24.6 MJ/kg (Table 2).

Table 2. Calorific value of dry biomass samples.

\begin{tabular}{ll}
\hline Biomass sample & Q, MJ/kg \\
\hline Cotton linter & 16.0 \\
Waste of textile & 16.2 \\
Used printing paper & 11.0 \\
Used newspaper & 12.9 \\
Used cardboard & 15.0 \\
Sawdust of wood & 18.1 \\
Bagasse & 17.0 \\
Corn stover & 14.5 \\
Wheat straw & 14.8 \\
Rice straw & 12.3 \\
Olive pomace & 21.2 \\
Residue of hydrolysis & 21.0 \\
Lignin Klason & 24.6 \\
\hline
\end{tabular}

The lowest calorific value, $11-13 \mathrm{MJ} / \mathrm{kg}$, was found for the samples having enhanced ash content, such as waste of printing and newsprint papers, and rice straw. The combustion heat of the other investigated biomass samples varied in the range from 15 to $18 \mathrm{MJ} / \mathrm{kg}$.

The calorific value of liquid biofuels and especially of biodiesel significantly exceeds the specific combustion heat of the solid plant materials (Table 3 ).

Table 3. Calorific value of liquid biofuels.

\begin{tabular}{ll}
\hline Liquid biofuel & Q, MJ/kg \\
\hline Bioethanol & 26.8 \\
Olive biodiesel & 37.2 \\
Modified biodiesel fuel & 37.4 \\
\hline
\end{tabular}

A forecast of the energy potential of various types of solid and liquid biofuels is import for theory and practice. For this purpose we checked the Mendeleev's equation (2) in order to predict the lower combustion heat of the samples.

$$
\mathrm{Q}(\mathrm{MJ} / \mathrm{kg})=0.339 \mathrm{C}+1.029 \mathrm{H}+0.109 \mathrm{~S}-0.109 \mathrm{O}
$$

where $\mathrm{C}, \mathrm{H}, \mathrm{S}, \mathrm{O}$ is percentage of the elements.

The investigations showed a good correlation between the calculated and experimental values (Fig. 1). It allows pre- 
dicting the combustion heat of different biomass samples and liquid biofuels with enough high confidence.

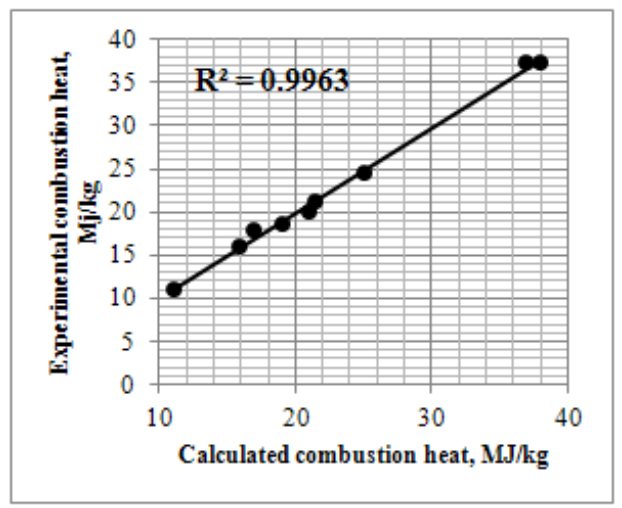

Fig. 1. Correlation between calculated and experimental combustion heats for the investigated samples.

At the discussion of the obtained results it is important to understand, which way of the biomass utilization might provide the greatest heat energy: direct burning of the solid biomass or burning of the liquid biofuel that was extracted from this biomass? To do this, it is advisable to analyze the some typical examples for production of the heat energy.

Example 1:

Pathway 1: One ton of fallen olive fruits (dry weight basis) containing $35 \%$ oil is burned.

Pathway 2: One ton of this biomass is squashed to obtain $25 \%$ of the vegetable oil, which is burned as a biodiesel.

\section{Example 2:}

Pathway 1: One ton of fallen olive fruits (dry weight basis) containing $35 \%$ oil is burned.

Pathway 2: One ton of this biomass is squashed to obtain $25 \%$ of the vegetable oil, which is subjected to transesterification (yield of 90\%), and the obtained biodiesel is burned.

Example 3:

Pathway 1: One ton of woody sawdust is burned.

Pathway 2: One ton of this biomass is pretreated with alkali (yield 70\%) and then is enzymatically converted into glucose (yield 50\%); the hydrolyzate is subjected to fermentation and distillation in order to produce the ethanol (yield of 90\%); and finally the obtained bioethanol is burned.

\section{Example 4:}

Pathway 1: One ton of the cotton waste (linter) is burned.

Pathway2: One ton of this biomass is enzymatically converted into glucose (yield 55\%); the hydrolyzate is subjected to fermentation and distillation in order to produce the ethanol (yield of 90\%); and finally the obtained bioethanol is burned.

\section{Example 5:}

Pathway 1: One ton of the pure cellulose is burned.

Pathway 2: One ton of this biomass is enzymatically converted into glucose with $100 \%$ conversion degree; the hydrolyzate is subjected to fermentation and distillation to produce the absolute ethanol, which is burned.

As it follows from the experiments, a most efficient way of the energy production is the direct burning of the plant biomass, while the burning of such amount of the liquid biofuel, which can be extracted from the plant material, gives a much smaller energetic effect (Table 4, Fig. 2).

Table 4. Heat energy of initial biomass and liquid biofuel*.

\begin{tabular}{llll}
\hline Example & $\mathbf{Q}_{\mathbf{b}}, \mathbf{G J}$ & $\mathbf{M}_{\mathbf{l f}}, \mathbf{k g}$ & $\mathbf{Q}_{\mathbf{l f}}, \mathbf{G J}$ \\
\hline 1 & 25.5 & 269 & 10.0 \\
2 & 25.5 & 246 & 9.2 \\
3 & 18.1 & 170 & 4.5 \\
4 & 16.0 & 242 & 6.5 \\
5 & 16.3 & 567 & 15.5 \\
\hline
\end{tabular}

*Note: $\boldsymbol{Q}_{b}, \boldsymbol{Q}_{l f}$ is a heat energy for $\mathbf{1}$ of the initial biomass, and for $\boldsymbol{M}_{\mathbf{l f}} \boldsymbol{k g}$ of the liquid biofuel, accordingly.

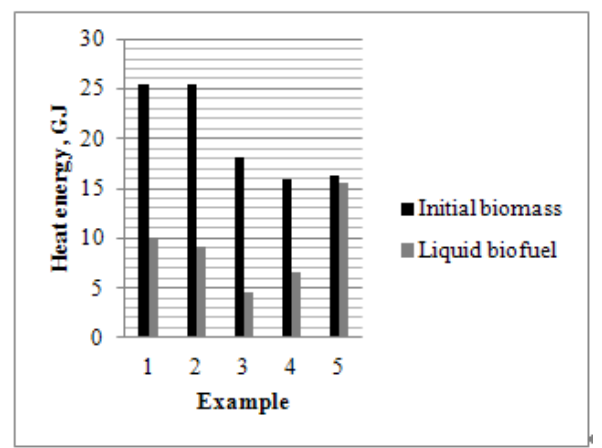

Fig. 2. Heat energy generated by burning of 1 ton of the initial biomass and of the obtained amount of the liquid biofuel.

Even in the theoretical example (5) the heat energy of the obtained amount of the bioethanol $(567 \mathrm{~kg})$ was lesser than the heat energy of one ton of the initial cellulose (Fig. 2).

Thus, despite the fact that the specific combustion heat of the liquid biofuel was higher than of the initial biomass, the total amount of the heat energy generated by burning of the biofuel was lesser due to the its limited yield from the plant material. Even the additional burning of solid residue of the biomass cannot cover the deficit in the heat energy. In fact, this deficit is much higher, because the calculations didn't include energy consumption for the production of biofuels, drying of the final products, and other expenses of the real production process.

\section{Conclusion}

The specific combustion heat of various types of biomasses and liquid biofuels were determined. The calorific value of the liquid biofuels was higher than of $\mathrm{f}$ the solid biomass samples. Despite of this fact, a most efficient way of the energy production is the direct burning of the plant biomass, while the burning of such amount of liquid biofuel, which can be obtained from plant material, gives a much smaller energetic effect.

In order to the production of the heat energy from biodiesel was energetically efficient, the yield of the vegetable oil should be $685 \mathrm{~kg}$ per 1 ton of the plant culture at least. However, this yield cannot be achieved in the practice be- 
cause the existing oil-containing cultures might generate much smaller oil volume, from 200 to $400 \mathrm{~kg}$ from 1 ton of the plant.

To provide the efficient energy production from bioethanol, the yield of this biofuel from 1 ton of biomass should be about $600 \mathrm{~kg}$. However, it is impossible because such amount of bioethanol exceeds its theoretical yield from the biomass.

The widespread use of the liquid biofuels is limited also due to their increased cost. However, if the liquid products are not aimed for burning, but will be applied as expensive organic solvents, medical or cosmetic remedies and the like, their production from the plant biomass would be cost effective.

\section{References}

[1] M. Ioelovich, "Biofuels - technology, problems and perspectives, a review," Journal SITA,. vol. 12, pp. 106-126, 2010.

[2] D. Klemm., B. Heublein, H.-P. Fink and A. Bohn, "Cellulose: fascinating biopolymer and sustainable raw material," Angew.
Chem., vol. 44, pp. 2 -37, 2005.

[3] K. A. Hossain, "Global energy consumption pattern and GDP," Int J Renewable Energy Technol. Res., vol. 1, pp. 23 - 29, 2012.

[4] D. Bacovsky, M. Dallos and M. Wörgetter, "Status of 2nd generation biofuels demonstration facilities," A report to IEA bioenergy task, n. 39, pp. 1-126, 2010.

[5] A. Obolenskaya, Z. Elnitskaya and A. Leonovich, Practical Guide for Chemistry of Wood and Cellulose, Moscow, 1991.

[6] H. Fukuda, A. Kondo and H. Noda,"Biodiesel fuel production by transesterification of oils," J. Biosci. Bioeng, vol. 92, pp. 405-416, 2001.

[7] M. Ioelovich and E. Morag, "Study of enzymatic hydrolysis of mild pretreated lignocellulosic biomasses," BioResources, vol. 7, pp. 1040-1052, 2012.

[8] M. Ioelovich and E. Morag, "Study of enzymatic hydrolysis of pretreated biomasses at increased solids loading," BioResources, vol. 7, pp. 4672-4682, 2012.

[9] T. D'Amore, I. Russell and G. Stewart, "Sugar utilization by yeast during fermentation,” J. Ind. Microbiology, vol. 4, pp. 315-324, 1989. 